\title{
Urinary function in elderly people with and without leukoaraiosis: relation to cognitive and gait function
}

\author{
Ryuji Sakakibara, Takamichi Hattori, Tomoyuki Uchiyama, Tomonori Yamanishi
}

\begin{abstract}
Objectives-To investigate urinary function in the elderly with and without white matter lesion (leukoaraiosis) in relation to cognitive and gait function.

Methods-Sixty three subjects were examined, with mean age 73 (range 62 to 86 years). Subjects with brainstem stroke or with large hemispheric lesions were excluded. Spin echo 1.5 T MRI images were graded from 0 to 4 for severity of white matter lesions. Urinary function was assessed by detailed questionnaire and urodynamic studies were performed in 33 of the subjects, including measurement of postmicturition residuals, water cystometry, and sphincter EMG. A mini mental state examination (MMSE) and examination of gait was also performed and compared with urinary function.

Results-Urodynamic studies showed subjects with grade 1-4 white matter lesions to have detrusor hyperreflexia more commonly $(82 \%)$ than those with grade 0 white matter lesions $(9 \%) \quad(p<0.05)$, indicating that leukoaraiosis was a factor associated with geriatric urinary dysfunction. Postmicturition residuals, low compliance, detrusor-sphincter dyssynergia, and uninhibited sphincter relaxation were also more common in grade 1-4 than in grade 0 white matter lesions, though the difference was not significant. In grade 1 white matter lesions urinary dysfunction (urge urinary incontinence) was more common than cognitive $(M M S E<19) \quad(p<0.05)$ and gait disorders (slowness, short step/festination, and loss of postural reflex) $(p<0.05)$, which increased together with the grade of white matter lesions $(p<0.05)$.
\end{abstract}

Conclusions-Urinary dysfunction is common and probably the early sign in elderly people with leukoaraiosis on MRI. (F Neurol Neurosurg Psychiatry 1999;67:658-660)

Keywords: geriatric urinary incontinence; leukoaraiosis; autonomic dysfunction; urodynamic study; detrusor hyperreflexia

The definite cause of high incidence of nocturnal urinary frequency and urge incontinence in elderly people is not known. ${ }^{1}$ Sleep disorder, nocturnal polyuria, and benign outlet obstruction of the lower urinary tract are possibly related to this condition. Detrusor hyperreflexia is a recognised finding in urodynamic study, which indicates central aetiology of this condition as well. ${ }^{23}$ Brain MRI showed frequent white matter signal abnormality (leukoaraiosis) in the brain of elderly people, ${ }^{4}$ some of which were considered as normal but others reflected ischaemic pathology. ${ }^{5}$ Leukoaraiosis in elderly people is relevant to cognitive disorder (multiinfarct dementia) ${ }^{78}$ and gait disorder. ${ }^{9}$ However, urinary dysfunction has not been systematically investigated. The present study aimed at evaluating urinary function in elderly people with and without leukoaraiosis in relation to cognitive and gait function.

\section{Subjects and methods}

We investigated 63 subjects, 28 men and 35 women, mean age 73 years, range $62-86$ years. The clinical backgrounds of these subjects were various, and 19 of them complained of symptoms relevant to leukoaraiosis, including gait difficulty in seven, dysarthria in five, and amnesia in five, and none was bed-ridden. Eleven had a history of a single lacunar infarct, including mild hemiparesis or hemisensory disturbance. Other subjects had tension-type headache, vestibular neuronitis, benign paroxysmal positional vertigo, Bell's palsy, or neurosis.

Brain MRI was performed on a General Electric imaging unit with a superconductive magnet to generate a field strength of $1.5 \mathrm{~T}$. Image acquisition was performed with a spin echo technique, the pulse sequences being repetition time (TR) 600-800 ms/echo time (TE) 20-25 for T1 weighted and TR 1500-2500 $\mathrm{ms} / \mathrm{TE} 25-120 \mathrm{~ms}$ for T2 weighted images. Sagittal, axial, and coronal scans were available for most subjects. The MRI studies were graded for the extent and severity of white matter lesions on a $0-4$ scale according to Brant-Zawadzki et $a l^{8}$ (fig 1). Grade 0 was assigned when no such lesions were evident. Grade 1 indicated punctated foci of high signal intensity in the white matter immediately at the top of the frontal horns of the lateral ventricles. Grade 2 was assigned when the white matter lesions were seen elsewhere but remained confined to the immediate subependymal region of the ventricles. Grade 3 indicated periventricu- and in revised form

2 March 1999

Accepted 7 April 1999 


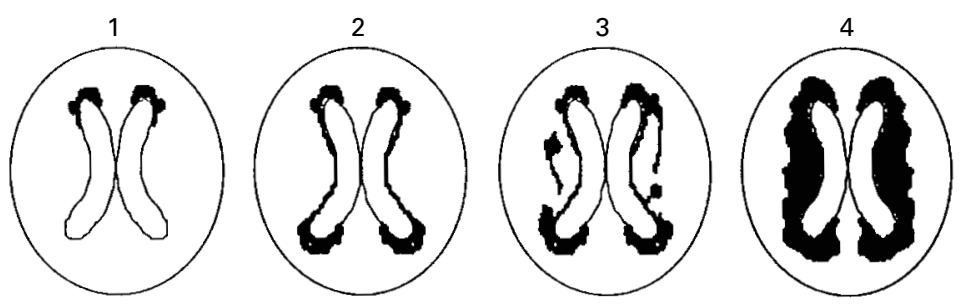

Figure 1 Schematic presentation of the grading of white matter lesion on MRI (according to Brand-Zawadzki et al $l^{8}$ ) Grade 1: punctated foci of high signal intensity in the white matter immediately at the top of the frontal horns of the lateral ventricles. Grade 2: white matter lesions were seen elsewhere but remained confined to the immediate subependymal region of the ventricles. Grade 3: periventricular as well as separate, discrete, deep white matter foci of signal abnormality. Grade 4: discrete white matter foci had become large and coalescent.

lar as well as separate, discrete, deep white matter foci of signal abnormality. Grade 4 was reserved for those cases in which the discrete white matter foci had become large and coalescent. Subjects with brainstem stroke or large hemispheric lesions were excluded from the study. According to these criteria, subjects were divided into five groups with almost the same age (grade 0,11 subjects, four men and seven women, mean age 73 years; grade 1, 15 subjects, five men and 10 women, mean age 74 years; grade 2, 12 subjects, eight men and four women, mean age 70 years; grade 3, 14 subjects, seven men and seven women, mean

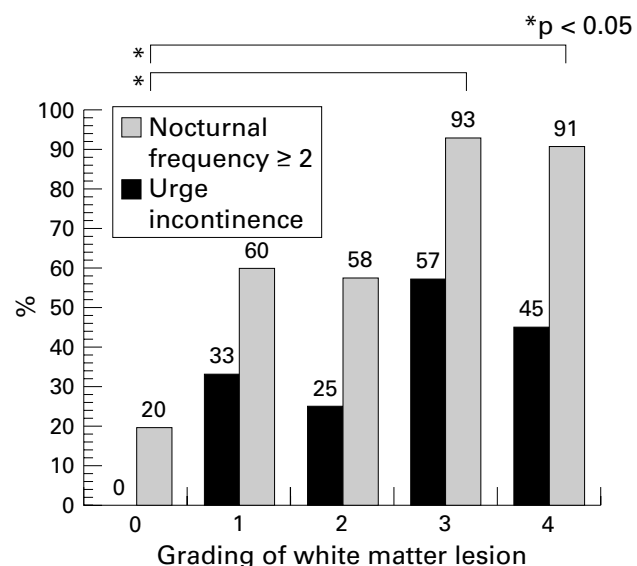

Figure 2 Urinary dysfunction and white matter lesion on $M R I$.

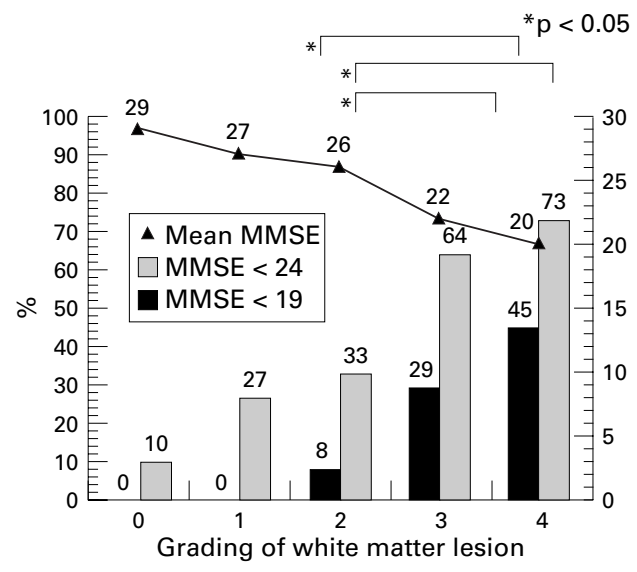

Figure 3 Cognitive disorder and white matter lesion on MRI. MMSE=mini mental state examination. age 75 years; grade 4,11 subjects, four men and seven women, mean age 74 years).

Urinary function was examined with a questionnaire and urodynamic studies. The questionnaire was answered by face to face interviewing, or taken from families of patients with cognitive disorder. Urinary dysfunction was graded as mild with nocturnal urinary frequency $\geqslant 2$ and severe with urge urinary incontinence. Urodynamic studies were performed in 33 of the subjects, divided into two groups according to the above criteria (grade 0 , all 11 elderly subjects as described above, two with nocturnal urinary frequency $\geqslant 2$ and none with urge urinary incontinence; grade 1-4, 22 subjects, 14 men and eight women, mean age 72 years, 19 with nocturnal urinary frequency $\geqslant 2$ and 12 with urge urinary incontinence). Urodynamic studies consisted of measurement of postmicturition residuals (normal value $<30$ $\mathrm{ml}$ ), medium fill water cystometry, and external sphincter EMG. The methods and definitions used for the urodynamic studies conformed to the standards proposed by the International Continence Society. ${ }^{10}$ Patients with urinary symptoms did not have an apparent urinary tract infection. Informed consent was obtained from all the patients before the urodynamic studies were made.

To compare with urinary function, we performed a mini mental state examination $(\mathrm{MMSE})^{11}$ and examination of gait. Cognitive disorder was graded as mild with score $<24$ and severe with score $<19$ on MMSE. The mean value of MMSE in each grade was also obtained. Apparent Alzheimer's disease or other neurodegenerative dementia was excluded from the study according to the diagnostic criteria in the DSM III (Diagnostic and Statistical Manual of Mental Disorders). ${ }^{12}$ Gait disorder was graded as mild with one feature and severe with three features of slowness of gait, short step/festination, and loss of postural reflex. Patients with Parkinson's disease or other degenerative parkinsonism were excluded from the study. Statistics were performed by Student's $t$ test.

\section{Results}

Urodynamic studies showed subjects with grade 1-4 white matter lesions to have detrusor hyperreflexia more commonly (82\%) than those with grade 0 white matter lesion (9\%) $(\mathrm{p}<0.05)$. Postmicturition residuals, low compliance, detrusor-sphincter dyssynergia, and uninhibited sphincter relaxation were also more common in grade 1-4 than in grade 0 white matter lesion, although there was no statistical difference. In subjects with grade 0 white matter lesion, urinary, cognitive, and gait disorders were found to be minimal, and these increased together with the grade of white matter lesion $(\mathrm{p}<0.05$, figs $2,3,4)$. In each grade of white matter lesion, urinary dysfunction was more common than cognitive and gait disorders, although there was no statistical significance except for severe urinary dysfunction (urge urinary incontinence), which was more 


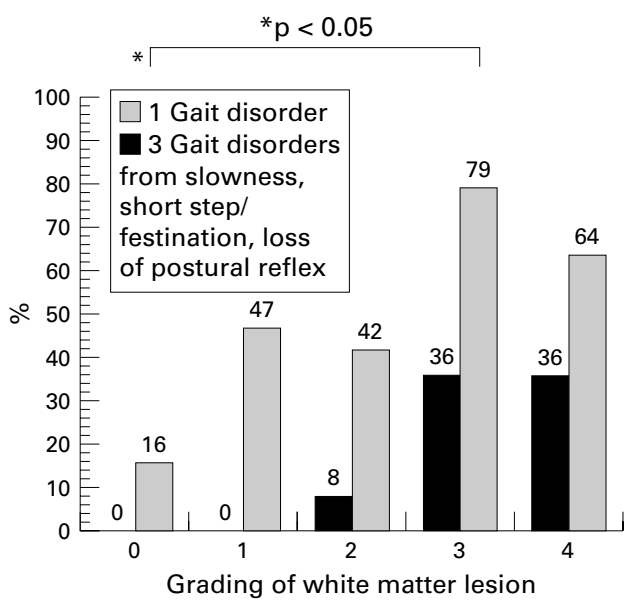

Figure 4 Gait disorder and white matter lesion on MRI.

common than severe cognitive $(p<0.05)$ and gait $(\mathrm{p}<0.05)$ disorders in grade 1 white matter lesion.

\section{Discussion}

The present study showed the incidence of detrusor hyperreflexia to be clearly different between grade $0(9 \%)$ and grade $1-4(82 \%)$ white matter lesions in groups of subjects with almost the same age. This finding indicates that leukoaraiosis is a factor involved in geriatric urinary dysfunction. The frequency of detrusor hyperreflexia in the present study was more common than the $41 \%-50 \%$ in Parkinson's disease $\mathrm{e}^{1314}$ and $40 \%$ in Alzheimer's disease, ${ }^{15}$ and as common as the $37 \%-82 \%$ found in acute hemispheric stroke, ${ }^{16}{ }^{17}$ and the $52 \%-100 \%$ in multiple system atrophy, ${ }^{18}{ }^{19}$ showing leukoaraiosis to be a major cause of central pelvic nerve dysfunction innervating the bladder. In the clinical context detrusor hyperreflexia appears as nocturnal urinary frequency, and then urge urinary incontinence. ${ }^{17}{ }^{18}$ Our previous data on acute hemispheric stroke showed the predominant lesions of urinary dysfunction in the anteromedial surface of the frontal lobe, anterior edge of the paraventricular white matter, and the genu of the internal capsule. ${ }^{17}$ The areas include the frontal micturition centre, proposed by Andrew and Nathan, ${ }^{20}$ and their descending pathways, as detected by reduced cerebral blood flow in a SPECT study of incontinent elderly people, ${ }^{21}$ which are probably involved in leukoaraiosis as well.

The present study showed urinary dysfunction (nocturnal urinary frequency/urge incontinence) to be more common than cognitive and gait disorders, particularly in subjects with grade 1 white matter lesions. This finding indicates that some elderly subjects with leukoaraiosis may have urinary dysfunction first, without any other neurological abnormalities.
The reason for this is not certain, but probable neuronal vulnerability associated with transmitters may have a role. Unlike acute stroke, the onset of leukoaraiosis seems insidious and its progression is not only stepwise but also gradual. Our data also suggest that there are still subjects with "silent" white matter lesions. ${ }^{22}$ However, urinary dysfunction seems to be common and probably the early sign in subjects with leukoaraiosis, which contributes to early suspicion of this disorder.

This study was supported in part by the grants of Japan Foundation of Ageing and Health, Ministry of Education, and Research Committee of Ataxic Diseases in the Ministry of Health and Welfare in Japan.

1 Williams ME, Pannill FC. Urinary incontinence in the eldrly; physiology, pathophysiology, diagnosis, and treatment. Ann Intern Med 1982;97:895-907.

2 Resnick NM, Yalla SV. Detrusor hyperactivity with impaired contractile function; an unrecognized but common cause of incontinence in elderly patients. $\mathcal{F} A M A 1987 ; 257: 3076-$ 81.

3 Kitada S, Ikei Y, Hasui Y, et al. Bladder function in elderly men with subclinical brain magnetic resonance imaging studies. F Urol 1992;147:1507-9.

4 Hachinski VC, Potter P, Merskey H. Leuko-araiosis. Arch Neurol 1987;44:21-3.

5 Fazekas F, Kleinert R, Offenbacher H, et al. Pathologic correlates of incidental MRI white matter signal hyperintensities. Neurology 1993;43:1683-9.

6 Baloh RW, Yue Q, Socotch TM, et al. White matter lesions and dysequilibrium in older people; 2 . clinicopathological correlation. Arch Neurol 1995;52:975-81.

7 Kotsuoris H, Barclay LL, Kheyfets S, et al. Urinary and gait disturbances as markers for early multi-infarct dementia. Stroke 1987;18:138-41.

8 Brant-Zawadzki M, Fein G, Van Dyke C, et al. MR imaging of the aging brain; pacthy white-matter lesions and dementia. AfNR Am $\mathcal{F}$ Neuroradiol 1985;6:675-82.

9 Baloh RW, Yue Q, Socotch TM, et al. White matter lesions and dysequilibrium in older people; 1. case-control comparison. Arch Neurol 1995;52:970-74.

10 Abrams P, Blaivas JG, Stanton SL, et al. The standardization of terminology of lower urinary tract function; produced by the International Continence Society Committee on Standardization of Terminology. World F Urol 1989;6:23345.

11 Folstein M, Folstein S, McHugh PR. Mini-mental state; a practical method for grading the cognitive state of patients for the clinician. F Psychiatr Res 1975;12:189-98.

12 American Psychiatric Association. Diagnostic and statistical manual of mental disorders, 3rd ed, revised. Washington, DC: American Psychiatric Press, 1987.

13 Andersen JT, Hebjorn S, Frimodt-Moller C, et al. Disturbance of micturition in Parkinson's disease. Acta Neurol Scand 1976;53:161-70

14 Hattori T, Yasuda K, Kita K, et al. Voiding dysfunction in Parkinson's disease. Fapanese fournal of Psychiatry and Neurology 1992;46:181-6. (In English.)

15 Sugiyama $\mathrm{T}$, Hashimoto $\mathrm{K}$, Kiwamoto $\mathrm{H}$, et al. Urinary incontinence in senile dementia of the Alzheimer type (SDAT). Int $\mathcal{F}$ Urol 1994;1:337-40.

16 Reding MJ, Winter SW, Hochrein SA, et al. Urinary incontinence after unilateral hemispheric stroke; a neurologictinence after unilateral hemispheric stroke; a neurologic-

17 Sakakibara R, Hattori T, Yasuda K, et al. Micturitional disturbance after acute hemispheric stroke; analysis of lesion site by CT and MRI. F Neurol Sci 1996;137:47-56.

18 Beck RO, Betts CD, Fowler CJ. Genitourinary dysfunction in multiple system atrophy; clinical features and treatment in 62 cases. $\mathcal{F}$ Urol $1994 ; 151: 1336-41$.

19 Sakakibara R, Hattori T, Tojo M, et al. Micturitional disturbance in multiple system atrophy. Fapanese fournal of Psychiatry and Neurology 1993;47:591-8. (In English.)

20 Andrew J, Nathan PW. Lesions of the anterior frontal lobes and disturbances of micturition and defaecation. Brain 1964;87:233-62.

21 Griffiths DJ, McCracken PN, Harrison GM, et al. Cerebral etiology of urinary urge incontinence in elderly people. Age Ageing 1994:23:246-50.

22 Kase CS, Wolf PA, Chodosh EH, et al. Prevalence of silent stroke in patients with initial stroke; the Framingham study. Stroke 1989;20:850-2. 